\title{
Esquema de discretização Flux-Spline aplicado à secagem, em meio poroso capilar
}

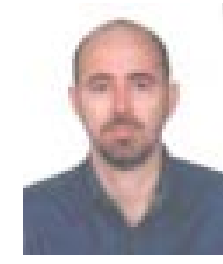

Paulo C. Oliveira ${ }^{1} \&$ José L. Lima²

1 CCA/UFES. CP 16, CEP 29500-000, Alegre, ES. E-mail: pacol@npd.ufes.br (Foto)

2 Bolsista de PIBIC - Agronomia, CCA/UFES. E-mail: lima-I@bol.com.br

Protocolo 118 - 21/8/2002 - Aprovado em 20/2/2003

\begin{abstract}
Resumo: Este trabalho foi desenvolvido com o objetivo de se apresentar a aplicação de um esquema de discretização mais eficiente para volumes finitos, denominado Flux-Spline utilizandose, para tal, de dois casos de transporte difusivo de umidade e calor, através de um meio poroso capilar. Os resultados da solução numérica do sistema de equações formado pelas equações de Luikov mostram desempenho adequado do esquema para este tipo de problema, quando comparado ao tradicional esquema de diferença central e ao método da transformada integral.
\end{abstract}

Palavras-chave: difusão, equações de Luikov, volumes finitos

\section{Flux-Spline discretization scheme applied to drying in capillary porous media}

\begin{abstract}
This study was conducted with the objective to present a more efficient discretization scheme to finite volumes method called Flux-Spline, utilizing for the purpose, two cases of pure diffusion in capillary porous media. The results of numerical simulation of the system of equations formed by Luikov equations showed a good performance of the scheme in comparison to the Central Difference Scheme and Generalized Integral Transform Technique method.
\end{abstract}

Key words: diffusion, Luikov equations, finite volumes

\section{INTRODUÇÃO}

Problemas práticos envolvendo transporte de calor e massa aparecem em processos como fusão e solidificação de materiais, secagem de madeira, armazenamento e beneficiamento de grãos, movimentação de água, poluentes ou adubos em solos, conforto ambiental em edificações, escoamentos atmosféricos e previsão de tempo. O método de volumes finitos é utilizado por grande parte da literatura dos problemas citados acima.

Dentre os esquemas de discretização usados em volumes finitos para difusão pura, o esquema de Diferença Central, descrito em Patankar (1980), gerado a partir da hipótese de que o fluxo difusivo da variável dependente é constante entre dois pontos da discretização, ocupa lugar de destaque na comunidade de simulação numérica, devido à sua simplicidade e aos bons resultados para este tipo de fenômeno. A fim de reduzir esforço computacional, Varejão (1979) demonstrou que, admitir uma variação linear do fluxo total da variável dependente transportada dentro de cada volume de controle, gera um perfil interpolante denominado Flux-Spline, que produz, para todos os problemas teste usados ao longo do seu trabalho, erros expressivamente menores que o esquema de Diferença Central. Nieckele (1985) confirmou o bom desempenho do esquema Flux-
Spline, comparando-o aos demais esquemas utilizados na literatura de métodos numéricos da época. Oliveira (1997) utilizou este esquema para simular convecção natural em cavidades abertas, também com bons resultados. A única modificação presente no esquema Flux-Spline, com relação ao tradicional esquema de diferença central, reside no aparecimento de mais um termo-fonte, cujo cálculo não possui qualquer dificuldade.

O presente trabalho teve como objetivo apresentar um esquema de discretização mais elaborado em relação aos atualmente utilizados pelos usuários deste método, para tratar problemas difusivos governados pelo sistema de equações descritas em Luikov \& Mikhailov (1965), Luikov (1966) e Luikov (1975). Tais equações, denominadas equações de Luikov, governam a transferência simultânea de calor e massa em problemas como a secagem de madeira, solos, cerâmicas e produtos agrícolas e farmacêuticos.

\section{MATERIAL E MÉTODOS}

\section{Discretização de difusão em regime permanente por volumes finitos}

A equação diferencial que rege o transporte de um escalar, $\phi$, em regime permanente, é: 


$$
\nabla \overrightarrow{\mathbf{J}}^{\phi}=\mathrm{S}^{\phi}
$$

em que o fluxo difusivo da variável dependente $\phi$ é $\mathrm{J}^{\phi}=-\Gamma \phi \nabla \phi$ e $\mathrm{S} \phi=\mathrm{Sc} \phi+\mathrm{Sp} \phi \phi$ representa o termo fonte linearizado como em Patankar (1980). A Eq. 1 pode ser colocada em coordenadas cartesianas na forma:

$$
\frac{\partial \mathrm{Jx}^{\phi}}{\partial \mathrm{X}}+\frac{\partial \mathrm{Jy}^{\phi}}{\partial \mathrm{Y}}+\frac{\partial \mathrm{Jz}^{\phi}}{\partial \mathrm{Z}}=\mathrm{S}^{\phi}
$$

O fluxo difusivo na direção X da variável dependente $\phi$ é:

$$
\mathrm{JX}^{\phi}=-\Gamma^{\phi} \frac{\partial \phi}{\partial \mathrm{X}}
$$

em que $\Gamma^{\phi}$ é o coeficiente de difusão, propriedade que expressa a capacidade do meio de transportar a variável dependente $\phi$. Num caso unidimensional, admite-se que o fluxo total e suas derivadas sejam posicionados nas faces dos volumes de controle, como mostrado na Figura 1. Assume-se que o fluxo total $\mathrm{JX}^{\phi}$ possa variar na direção $\mathrm{X}_{\mathrm{i}}$, de forma linear, ao longo de cada volume de controle. Desta hipótese obtém-se a seguinte expressão para o volume de controle i:

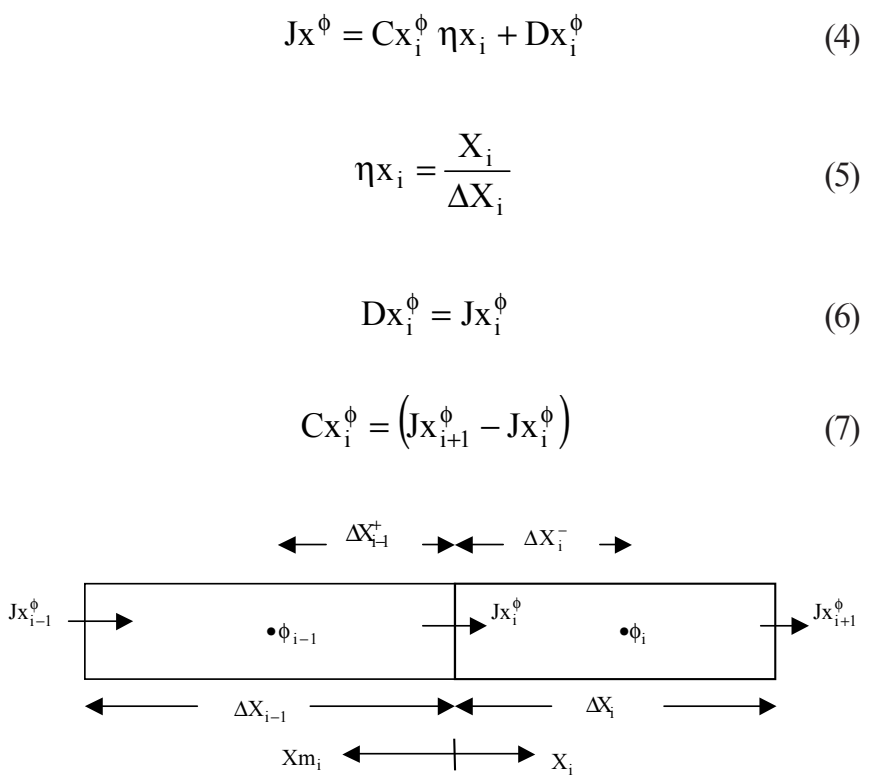

Figura 1. Discretização unidimensional para volumes finitos

Para o volume de controle (i-1) mostrado na Figura 1 sob a coordenada $\mathrm{Xm}_{\mathrm{i}}$ :

$$
\mathrm{Jxm}^{\phi}=\mathrm{Cxm}_{\mathrm{i}}^{\phi} \eta \mathrm{xm}_{\mathrm{i}}+\mathrm{Dxm}_{\mathrm{i}}^{\phi}
$$

em que:

$$
\eta \times m_{i}=\frac{X m_{i}}{\Delta X_{i-1}}
$$

$$
\operatorname{Dxm}_{\mathrm{i}}=-\mathrm{Jx}_{\mathrm{i}}^{\phi}
$$

$$
\mathrm{Cxm}_{\mathrm{i}}^{\phi}=\mathrm{Jx}_{\mathrm{i}}^{\phi}-\mathrm{JX}_{\mathrm{i}-1}^{\phi}
$$

Obtém-se, da igualdade entre as Eqs. (3) e (4) para um volume de controle $\mathrm{i}$ :

$$
-\Gamma_{i}^{\phi} \frac{\mathrm{d} \phi}{d x_{i}}=C x_{i}^{\phi} \eta x_{i}+D x_{i}^{\phi}
$$

A solução da equação diferencial acima, assumindo-se o coeficiente $\Gamma_{i}^{\phi}$ constante dentro deste volume de controle, fornece o perfil interpolante do esquema de discretização para difusão pura:

$$
\phi=\frac{-1.0}{\Gamma_{i}^{\phi}}\left[\frac{1}{2} \mathrm{Cx}_{\mathrm{i}}^{\phi} \frac{\mathrm{X}_{\mathrm{i}}^{2}}{\Delta \mathrm{X}_{\mathrm{i}}}+\mathrm{Dx}_{\mathrm{i}}^{\phi} \mathrm{X}_{\mathrm{i}}\right]+\mathrm{C}_{\mathrm{vci}}^{\phi}
$$

Com a condição $\phi\left(\mathrm{X}_{\mathrm{i}}=\Delta \mathrm{X}_{\mathrm{i}}\right)=\phi_{\mathrm{i}}$, obtém-se o valor da constante de integração para o volume de controle $\mathrm{i}$ :

$$
\mathrm{C}_{\mathrm{vci}}^{\phi}=\left(\frac{\Delta \mathrm{X}_{\mathrm{i}}^{-}}{\Gamma_{\mathrm{i}}^{\phi}} \eta \mathrm{mx}_{\mathrm{i}} \frac{1}{2}\right) \mathrm{Cx}_{\mathrm{i}}^{\phi}+\mathrm{Dx}_{\mathrm{i}}^{\phi} \frac{\Delta \mathrm{X}_{\mathrm{i}}^{-}}{\Gamma_{\mathrm{i}}^{\phi}}+\phi_{\mathrm{i}}
$$

em que:

$$
\eta \mathrm{mx}_{\mathrm{i}}=\frac{\Delta \mathrm{X}_{\mathrm{i}}^{-}}{\Delta \mathrm{X}_{\mathrm{i}}}
$$

Para o volume de controle (i - 1) usando-se o sistema de coordenadas $\mathrm{Xm}_{\mathrm{i}}$ mostrado na Figura 1 , com a condição $\phi\left(\mathrm{Xm}_{\mathrm{i}}\right.$ $\left.=\Delta \mathrm{X}_{\mathrm{i}-1}\right)=\phi_{\mathrm{i}-1}$, tem-se uma equação similar a Eq. 14:

$\mathrm{C}_{\mathrm{vci}-1}^{\phi}=\left(\frac{\Delta \mathrm{X}_{\mathrm{i}-1}^{+}}{\Gamma_{\mathrm{i}-1}^{\phi}} \eta \mathrm{px}_{\mathrm{i}-1} \frac{1}{2}\right) \operatorname{Cxm}_{\mathrm{i}}^{\phi}+\operatorname{Dxm}_{\mathrm{i}}^{\phi} \frac{\Delta \mathrm{X}_{\mathrm{i}-1}^{+}}{\Gamma_{\mathrm{i}-1}^{\phi}}+\phi_{\mathrm{i}-1}$

em que:

$$
\eta \mathrm{px}_{\mathrm{i}-1}=\frac{\Delta \mathrm{X}_{\mathrm{i}-1}^{+}}{\Delta \mathrm{X}_{\mathrm{i}-1}}
$$

\section{Condição de continuidade para a variável dependente $\phi$}

$\mathrm{Na}$ interface entre os volumes de controle i e (i - 1) a variável dependente possui um valor único, ou seja:

$$
\phi\left(\mathrm{X}_{\mathrm{i}}=0\right)=\phi\left(\mathrm{Xm}_{\mathrm{i}}=0\right)
$$

O que equivale a: 


$$
\mathrm{C}_{\mathrm{vci}}^{\phi}=\mathrm{C}_{\mathrm{vci}-1}^{\phi}
$$

\section{Equação para os fluxos na direção $X$}

Após a substituição em (19) das Eqs. (14) e (16) obtém-se a expressão para os fluxos difusivos $\mathrm{Jx}_{\mathrm{i}} \phi$, ao longo do domínio emx:

$$
\mathrm{Jx}_{\mathrm{i}}^{\phi}=\mathrm{DJX}_{\mathrm{i}}^{\phi}\left(\phi_{\mathrm{i}-1}-\phi_{\mathrm{i}}\right)+\mathrm{Jhatx}_{\mathrm{i}}^{\phi}
$$

em que:

$$
\begin{gathered}
\mathrm{DJx}_{i}^{\phi}=\frac{1.0}{\left(\frac{\Delta \mathrm{X}_{\mathrm{i}}^{-}}{\Gamma_{\mathrm{i}}^{\phi}}+\frac{\Delta \mathrm{X}_{\mathrm{i}-1}^{+}}{\Gamma_{\mathrm{i}-1}^{\phi}}\right)} \\
\mathrm{Jhatx}_{\mathrm{i}}^{\phi}=\mathrm{BJx}_{\mathrm{i}}^{\phi}\left(\mathrm{Jx}_{\mathrm{i}}^{\phi}-\mathrm{Jx}_{\mathrm{i}+1}^{\phi}\right)-\mathrm{CJ} \mathrm{x}_{\mathrm{i}}^{\phi}\left(\mathrm{Jx}_{\mathrm{i}-1}^{\phi}-\mathrm{Jx}_{\mathrm{i}}^{\phi}\right) \\
\mathrm{BJx}_{\mathrm{i}}^{\phi}=\frac{1}{2} \mathrm{DJ} \mathrm{x}_{\mathrm{i}}^{\phi} \frac{\Delta \mathrm{X}_{\mathrm{i}}^{-}}{\Gamma_{\mathrm{i}}^{\phi}} \eta \mathrm{mx}_{\mathrm{i}} \\
\mathrm{CJx}_{\mathrm{i}}^{\phi}=\frac{1}{2} \mathrm{DJ} \mathrm{x}_{\mathrm{i}}^{\phi} \frac{\Delta \mathrm{X}_{\mathrm{i}-1}^{+}}{\Gamma_{\mathrm{i}-1}^{\phi}} \eta p \mathrm{x}_{\mathrm{i}-1}
\end{gathered}
$$

\section{Equação de discretização para a variável dependente $\phi$}

A discretização da equação de governo (2) para um caso tridimensional em regime permanente fornece, de acordo com o método de volumes finitos:

$$
\begin{aligned}
& \left(\mathrm{Jx}_{\mathrm{i}+1, \mathrm{j}, \mathrm{k}}^{\phi}-\mathrm{JX}_{\mathrm{i}, \mathrm{j}, \mathrm{k}}^{\phi}\right) \Delta \mathrm{Y}_{\mathrm{j}} \Delta \mathrm{Z}_{\mathrm{k}}+ \\
& \left(\mathrm{Jy}_{\mathrm{i}, \mathrm{j}+1, \mathrm{k}}^{\phi}-\mathrm{Jy}_{\mathrm{i}, \mathrm{j}, \mathrm{k}}\right) \Delta \mathrm{X}_{\mathrm{i}} \Delta \mathrm{Z}_{\mathrm{k}}+\left(\mathrm{JZ}_{\mathrm{i}, \mathrm{j}, \mathrm{k}+1}^{\phi}-\mathrm{JZ}_{\mathrm{i}, \mathrm{j}, \mathrm{k}}^{\phi}\right) \Delta \mathrm{X}_{\mathrm{i}} \Delta \mathrm{Y}_{\mathrm{j}}= \\
& \left(\mathrm{Sc}_{\mathrm{i}, \mathrm{j}, \mathrm{k}}^{\phi}+\mathrm{Sp}_{\mathrm{i}, \mathrm{j}, \mathrm{k}}^{\phi} \phi_{\mathrm{i}, \mathrm{j}, \mathrm{k}}\right)\left(\Delta \mathrm{V}_{\mathrm{i}, \mathrm{j}, \mathrm{k}}=\Delta \mathrm{X}_{\mathrm{i}} \Delta \mathrm{Y}_{\mathrm{j}} \Delta \mathrm{Z}_{\mathrm{k}}\right)
\end{aligned}
$$

Substituindo-se na expressão acima as equações para os fluxos, obter-se-á, a equação de discretização para a variável dependente $\phi$ :

$$
\begin{aligned}
\operatorname{AP}_{\mathrm{i}, \mathrm{j}, \mathrm{k}}^{\phi} \phi_{i, j, \mathrm{k}}= & \operatorname{AIM}_{\mathrm{i}, \mathrm{j}, \mathrm{k}}^{\phi} \phi_{\mathrm{i}-1, \mathrm{j}, \mathrm{k}}+\operatorname{AIP}_{\mathrm{i}, \mathrm{j}, \mathrm{k}}^{\phi} \phi_{i+1, \mathrm{j}, \mathrm{k}}+ \\
& \operatorname{AJM}_{\mathrm{i}, \mathrm{j}, \mathrm{k}}^{\phi} \phi_{\mathrm{i}, \mathrm{j}-1, \mathrm{k}}+\operatorname{AJP}_{\mathrm{i}, \mathrm{j}, \mathrm{k}}^{\phi} \phi_{i, j+1, \mathrm{k}}+ \\
& \operatorname{AKM}_{\mathrm{i}, \mathrm{j}, \mathrm{k}}^{\phi} \phi_{1, \mathrm{j}, \mathrm{k}-1}+\operatorname{AKP}_{\mathrm{i}, \mathrm{j}, \mathrm{k}}^{\phi} \phi_{i, j, \mathrm{k}+1}+\operatorname{CON}_{\mathrm{i}, \mathrm{j}, \mathrm{k}}^{\phi}
\end{aligned}
$$

em que:

$$
\begin{aligned}
& \operatorname{AIM}_{\mathrm{i}, \mathrm{j}, \mathrm{k}}^{\phi}=\mathrm{DJx}_{\mathrm{i}, \mathrm{j}, \mathrm{k}}^{\phi} \Delta \mathrm{Y}_{\mathrm{j}} \Delta \mathrm{Z}_{\mathrm{k}} \\
& \mathrm{AIP}_{\mathrm{i}, \mathrm{j}, \mathrm{k}}^{\phi}=\mathrm{DJx}_{\mathrm{i}+1, \mathrm{j}, \mathrm{k}}^{\phi} \Delta \mathrm{Y}_{\mathrm{j}} \Delta \mathrm{Z}_{\mathrm{k}}
\end{aligned}
$$

$$
\operatorname{AJM}_{\mathrm{i}, \mathrm{j}, \mathrm{k}}^{\phi}=\mathrm{DJy}_{\mathrm{i}, \mathrm{j}, \mathrm{k}}^{\phi} \Delta \mathrm{X}_{\mathrm{i}} \Delta \mathrm{Z}_{\mathrm{k}}
$$

$$
\mathrm{AJP}_{\mathrm{i}, \mathrm{j}, \mathrm{k}}^{\phi}=\mathrm{DJy}_{\mathrm{i}, \mathrm{j}+1, \mathrm{k}}^{\phi} \Delta \mathrm{X}_{\mathrm{i}} \Delta \mathrm{Z}_{\mathrm{k}}
$$

$$
\mathrm{AKM}_{\mathrm{i}, \mathrm{j}, \mathrm{k}}^{\phi}=\mathrm{DJZ}_{\mathrm{i}, \mathrm{j}, \mathrm{k}}^{\phi} \Delta \mathrm{X}_{\mathrm{i}} \Delta \mathrm{Y}_{\mathrm{j}}
$$

$$
\mathrm{AKP}_{\mathrm{i}, \mathrm{j}, \mathrm{k}}^{\phi}=\mathrm{DJ}_{\mathrm{i}, \mathrm{j}, \mathrm{k}+1}^{\phi} \Delta \mathrm{X}_{\mathrm{i}} \Delta \mathrm{Y}_{\mathrm{j}}
$$

$$
\begin{aligned}
& \mathrm{AP}_{\mathrm{i}, \mathrm{j}, \mathrm{k}}^{\phi}= \mathrm{AIM}_{\mathrm{i}, \mathrm{j}, \mathrm{k}}^{\phi}+\mathrm{AIP}_{\mathrm{i}, \mathrm{j}, \mathrm{k}}^{\phi}+\mathrm{AJM}_{\mathrm{i}, \mathrm{j}, \mathrm{k}}^{\phi}+ \\
& \mathrm{AJP}_{\mathrm{i}, \mathrm{j}, \mathrm{k}}+\mathrm{AKM}_{\mathrm{i}, \mathrm{j}, \mathrm{k}}^{\phi}+\mathrm{AKP}_{\mathrm{i}, \mathrm{j}, \mathrm{k}}^{\phi}- \mathrm{Sp}_{\mathrm{i}, \mathrm{j}, \mathrm{k}}^{\phi} \Delta \mathrm{X}_{\mathrm{i}} \Delta \mathrm{Y}_{\mathrm{j}} \Delta \mathrm{Z}_{\mathrm{k}} \\
& \mathrm{CON}_{\mathrm{i}, \mathrm{j}, \mathrm{k}}^{\phi}=\mathrm{Sc}_{\mathrm{i}, \mathrm{j}, \mathrm{k}}^{\phi} \Delta \mathrm{V}_{\mathrm{i}, \mathrm{j}, \mathrm{k}}-\operatorname{DIVhat}_{\mathrm{i}, \mathrm{j}, \mathrm{k}}^{\phi} \\
& \operatorname{DIVhat~}_{\mathrm{i}, \mathrm{j}, \mathrm{k}}^{\phi}=\left(\operatorname{Jhatx}_{\mathrm{i}+1, \mathrm{j}, \mathrm{k}}^{\phi}-\operatorname{Jhatx}_{\mathrm{i}, \mathrm{j}, \mathrm{k}}^{\phi}\right) \Delta \mathrm{Y}_{\mathrm{j}} \Delta \mathrm{Z}_{\mathrm{k}}+ \\
&\left(\operatorname{Jhaty}_{\mathrm{i}, \mathrm{j}+1, \mathrm{k}}^{\phi}-\operatorname{Jhaty}_{\mathrm{i}, \mathrm{j}, \mathrm{k}}\right) \Delta \mathrm{X}_{\mathrm{i}} \Delta \mathrm{Z}_{\mathrm{k}}+ \\
&\left(\operatorname{Jhatz}_{\mathrm{i}, \mathrm{j}, \mathrm{k}+1}^{\phi}-\operatorname{Jhatz}_{\mathrm{i}, \mathrm{j}, \mathrm{k}}^{\phi}\right) \Delta \mathrm{X}_{\mathrm{i}} \Delta \mathrm{Y}_{\mathrm{j}}
\end{aligned}
$$

\section{Discretização de problema difusivo transiente por volumes finitos}

A discretização proposta por Patankar (1980) para casos transientes, é totalmente implícita no tempo, o que faz com que se modifiquem as seguintes expressões:

$$
\begin{aligned}
\mathrm{AP}_{\mathrm{i}, \mathrm{j}, \mathrm{k}}^{\phi}= & \sum \mathrm{A}_{\mathrm{nb}}-\mathrm{Sp}_{\mathrm{i}, \mathrm{j}, \mathrm{k}}^{\phi} \Delta \mathrm{V}_{\mathrm{i}, \mathrm{j}, \mathrm{k}}+\frac{\Delta \mathrm{V}_{\mathrm{i}, \mathrm{j}, \mathrm{k}}}{\Delta \tau} \\
\mathrm{AP}_{\mathrm{i}, \mathrm{j}, \mathrm{k}}^{\phi} \phi_{\mathrm{i}, \mathrm{j}, \mathrm{k}}= & \sum \mathrm{A}_{\mathrm{nb}} \phi_{\mathrm{nb}}+\operatorname{Sc}_{\mathrm{i}, \mathrm{j}, \mathrm{k}}^{\phi} \Delta \mathrm{V}_{\mathrm{i}, \mathrm{j}, \mathrm{k}}+ \\
\phi_{\mathrm{i}, \mathrm{j}, \mathrm{k}}^{\text {old }} & \frac{\Delta \mathrm{V}_{\mathrm{i}, \mathrm{j}, \mathrm{k}}}{\Delta \tau}+\operatorname{Ssp}_{\mathrm{i}, \mathrm{j}, \mathrm{k}}^{\phi}
\end{aligned}
$$

em que $\Sigma \mathrm{A}_{\mathrm{nb}}$ é a somatória dos coeficientes vizinhos ao ponto de coordenadas ( $\mathrm{i}, \mathrm{j}, \mathrm{k}), \phi \mathrm{i}, \mathrm{j}, \mathrm{k}$ é o valor da variável dependente no tempo atual, $\phi \mathrm{i}, \mathrm{j}$, $\mathrm{k}$ é o valor da variável dependente no tempo anterior e $\Delta \tau$ é o intervalo de tempo adimensional. O método de solução do sistema de equações algébricas é descrito em detalhes por Oliveira (1999).

\section{RESULTADOS E DISCUSSÃO}

O esquema anteriormente descrito será aplicado a problemas transientes uni e bidimensionais. As expressões para a discretização unidimensional são obtidas suprimindo-se as direções Y e Z. As equações de Luikov (1965) adimensionalizadas, para problemas de secagem em corpos porosos 
capilares, sob condições de pressão constante, de acordo com Ribeiro et al. (1993) são:

$$
\begin{gathered}
\frac{\partial \theta_{1}}{\partial \tau}-\alpha \nabla^{2} \theta_{1}=-\beta \nabla^{2} \theta_{2} \\
\frac{\partial \theta_{2}}{\partial \tau}-\operatorname{Lu} \nabla^{2} \theta_{2}=-\operatorname{LuPn} \nabla^{2} \theta_{1}
\end{gathered}
$$

em que: $\theta_{1}=\left[\mathrm{T}(\mathrm{x}, \mathrm{y}, \mathrm{z}, \mathrm{t})-\mathrm{T}_{0}\right] / \mathrm{T}_{\mathrm{s}}-\mathrm{T}_{0}, \theta_{2}=\left[\mathrm{U}_{\mathrm{o}}-\mathrm{U}(\mathrm{x}, \mathrm{y}, \mathrm{z}, \mathrm{t})\right] / \mathrm{U}_{\mathrm{o}}-\mathrm{U}^{*}$, $\alpha=1+\varepsilon$ KoLuPn e $\beta=\varepsilon$ KoLu.

$\mathrm{T}_{0}$ e $\mathrm{U}_{0}$ são a temperatura e umidade iniciais do corpo poroso, $\mathrm{T}_{\mathrm{S}}$ é a temperatura do ar de secagem e $\mathrm{U}^{*}$ é a umidade de equilíbrio do corpo poroso com o ar.

Os parâmetros adimensionais restantes envolvidos, $\alpha, \beta, \varepsilon$, Lu, Ko e Pn, são definidos em Ribeiro et al. (1993). X $=X / L_{c}$ é a coordenada espacial adimensional e $\tau=\mathrm{at} / \mathrm{L}_{\mathrm{c}}{ }^{2}$ o tempo adimensional. A discretização do termo transiente é feita usando-se diferença finita de primeira ordem e as equações geradas são totalmente implícitas no tempo, como proposto por Patankar (1980). A malha espacial utilizada nos dois casos será acompanhada por uma malha de 50 passos, ao longo do tempo.

\section{Problema Teste 1}

Foi retirado de Ribeiro et al. (1993) e consiste numa placa porosa úmida de espessura unitária exposta ao ar seco, que sofre a ação de secagem devido a um fluxo de calor em sua face inferior, como mostrado na Figura 2. Os coeficientes das equações de transporte são constantes. O objetivo é obter-se a distribuição de temperatura e umidade para vários tempos, ao longo de $\mathrm{X}$.

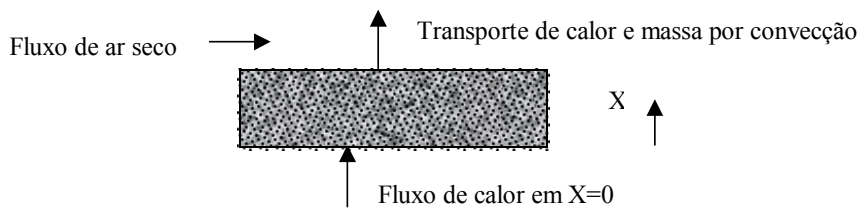

Figura 2. Secagem em placa porosa úmida aquecida em $X=0$

A condição inicial para a temperatura e umidade é descrita por:

$$
\begin{gathered}
\theta_{1}(X, \tau=0)=\theta_{2}(X, \tau=0)=0 \\
\frac{\partial \theta_{1}(0, \tau)}{\partial X}=-Q \\
\frac{\partial \theta_{2}(0, \tau)}{\partial X}-\operatorname{Pn} \frac{\partial \theta_{1}(0, \tau)}{\partial X}=0
\end{gathered}
$$

$\frac{\partial \theta_{1}}{\partial X}-\mathrm{Bi}_{\mathrm{q}}\left[1-\theta_{1}\right]+(1-\varepsilon) \operatorname{KoLuBi}_{\mathrm{m}}\left[1-\theta_{2}\right]=0$ para $\mathrm{X}=1(42)$

$$
-\frac{\partial \theta_{2}}{\partial X}+\operatorname{Pn} \frac{\partial \theta_{1}}{\partial X}+B i_{m}\left[1-\theta_{2}\right]=0 \quad \text { para } X=1
$$

$\mathrm{Bi}_{\mathrm{q}}$ é o coeficiente de transferência de calor adimensional, $\mathrm{Bi}_{\mathrm{m}} \mathrm{o}$ coeficiente de transferência de massa adimensional e $\mathrm{Q}$ o fluxo de calor adimensional em $\mathrm{X}$ igual a zero. Os valores dos parâmetros adimensionais usados, foram $\mathrm{Lu}=0,4, \mathrm{Ko}=5,0, \mathrm{Pn}$ $=0,6, \mathrm{Bi}_{\mathrm{q}}=\mathrm{Bi}_{\mathrm{m}}, \varepsilon=0,2 \mathrm{e} \mathrm{Q}=0,9$.

Os resultados da simulação numérica para temperatura e umidade, utilizando-se o esquema Flux-Spline com vinte volumes de controle, como funções da posição ao longo de X, para os tempos $\tau$ igual a $0,1,0,4$ e 0,8 , estão plotados na Figura 3 ,

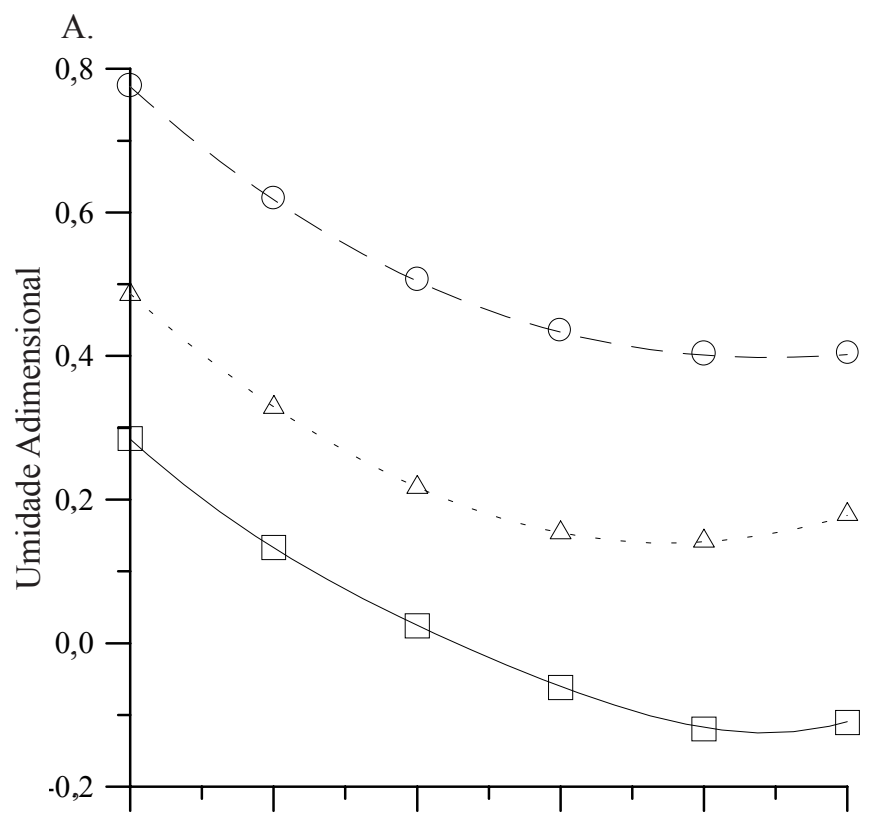

B.

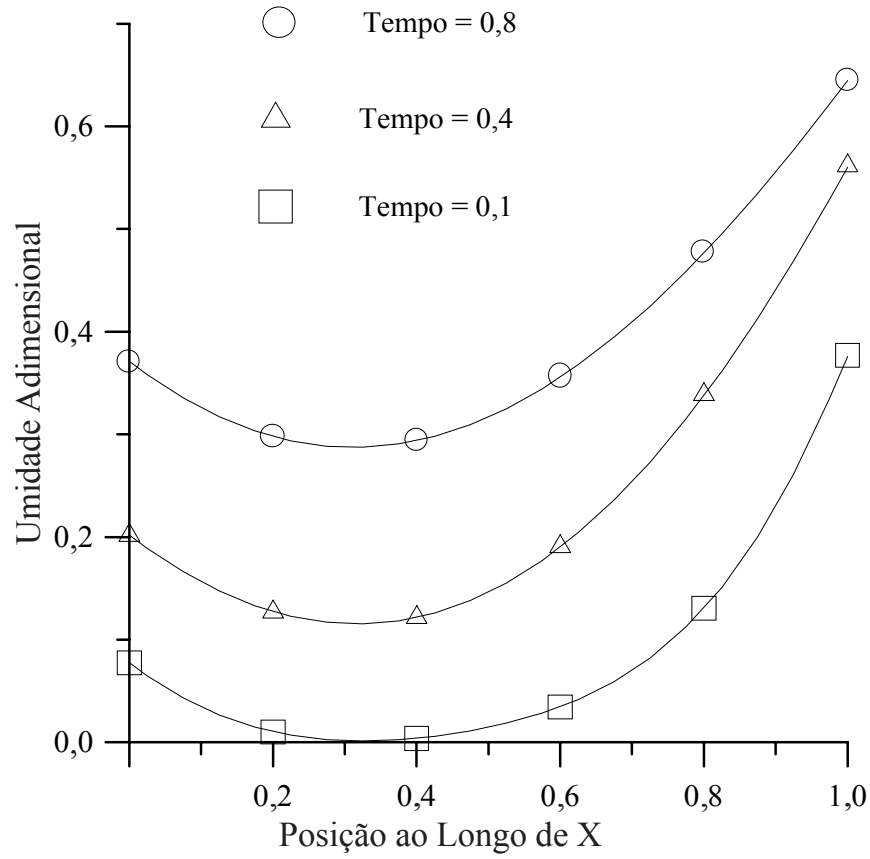

Figura 3. Perfis de temperatura (A) e umidade (B) adimensionais obtidos por Flux-Spline (linha cheia) com 20 vcs, comparadas com os resultados pontuais de Ribeiro et al. (1993) 
A.
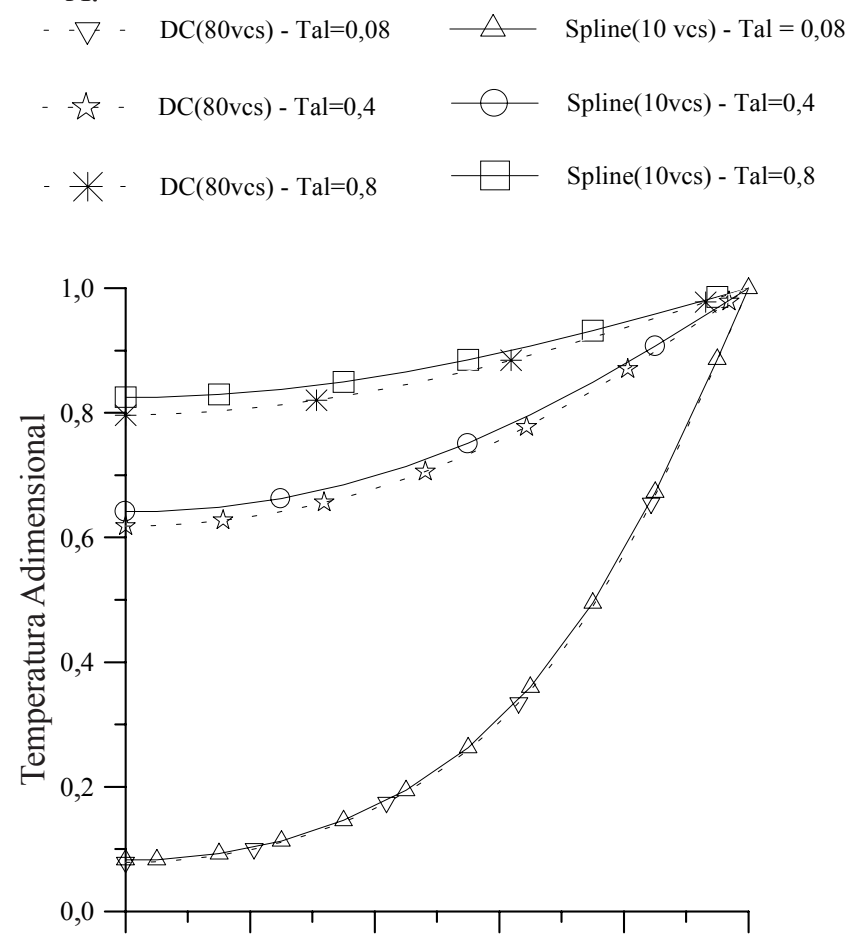

B.

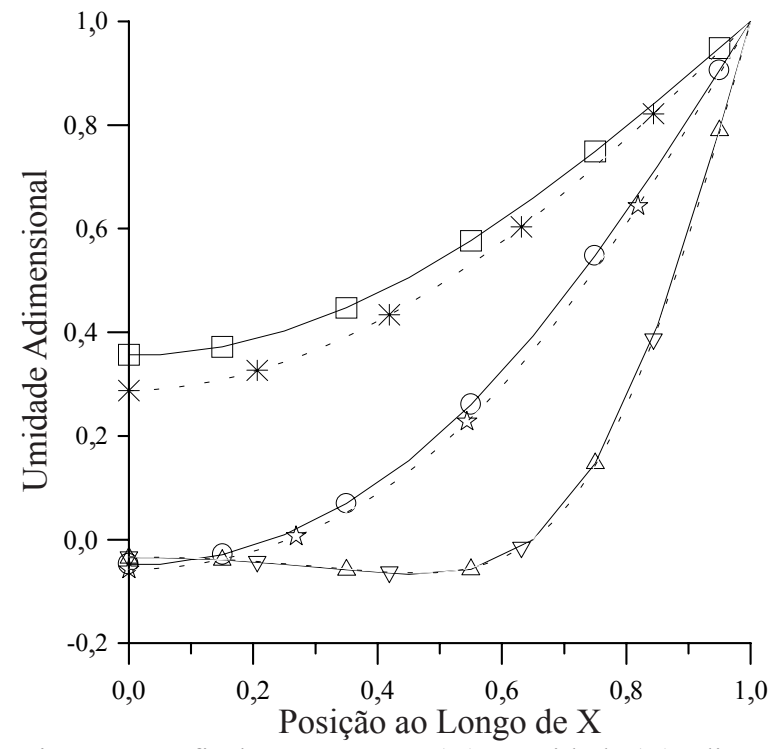

Figura 4. Perfis de temperatura (A) e umidade (B) adimensionais obtidos por Flux-Spline (10x10) comparados com solução usando-se Dif. Central com 80x80 ves

com linhas cheias. Os seis resultados pontuais de Ribeiro et al. (1993) obtidos por meio do método da transformada integral (usado como referência) são aqui representados pelos símbolos para cada tempo $\tau$. Nota-se excelente concordância com a solução de referência, devido ao fato do resultado com o método de volumes finitos passar pelos seis pontos, utilizando-se reduzido número de volumes de controle.

\section{Problema Teste 2}

Retirado de Ribeiro \& Lobo (1998), consiste numa placa porosa bidimensional de dimensões unitárias com coeficientes constantes. A face Oeste $(\mathrm{X}=0)$ e a face $\operatorname{Sul}(\mathrm{Y}=0)$ são isoladas em termos de fluxo de calor e umidade, de forma que calor e umidade fluem apenas pelas faces Oeste e Norte. Os valores dos parâmetros adimensionais são $\mathrm{Lu}=0,4, \mathrm{Ko}=3,0, \mathrm{Pn}=1,0$ e $\varepsilon=0,2$. As condições iniciais são as mesmas do caso anterior. Para $\tau>0$, as condições de contorno são:

$$
\begin{gathered}
\frac{\partial \theta_{1}(0, \mathrm{Y}, \tau)}{\partial \mathrm{X}}=\frac{\partial \theta_{1}(\mathrm{X}, 0, \tau)}{\partial \mathrm{Y}}=0 \\
\frac{\partial \theta_{2}(0, \mathrm{Y}, \tau)}{\partial \mathrm{X}}-\operatorname{Pn} \frac{\partial \theta_{1}(0, \mathrm{Y}, \tau)}{\partial \mathrm{X}}=0 \\
\frac{\partial \theta_{2}(\mathrm{X}, 0, \tau)}{\partial \mathrm{Y}}-\operatorname{Pn} \frac{\partial \theta_{1}(\mathrm{X}, 0, \tau)}{\partial \mathrm{Y}}=0 \\
\theta_{1}(1, \mathrm{Y}, \tau)=\theta_{2}(1, \mathrm{Y}, \tau)=1,0 \\
\theta_{1}(\mathrm{X}, 1, \tau)=\theta_{2}(\mathrm{X}, 1, \tau)=1,0
\end{gathered}
$$

As Figuras 4A e 4B exibem os resultados do esquema FluxSpline (linha cheia) para temperatura e umidade para $Y=0$, ao longo da coordenada $X$, para três tempos adimensionais $\tau(0,08$, 0,4 e 0,8 ) em comparação com uma solução de referência (linha tracejada) obtida usando-se o tradicional esquema de Diferença Central com uma malha de $80 \times 80$ volumes de controle. Note que a aproximação dos resultados tomados como referência é expressiva no sentido de demonstrar a eficiência do esquema Flux-Spline operando com apenas 10x10 volumes de controle.

\section{CONCLUSÕES}

1. O esquema Flux-Spline aqui descrito mostrou, com malhas relativamente grosseiras e através dos gráficos apresentados, resultados adequados de precisão para o caso de difusão pura em meio poroso capilar governado pelas equações de Luikov, tanto no caso unidimensional como no bidimensional, tendo em vista as comparações com o método da transformada integral no primeiro problema teste e o esquema de diferença central no segundo.

2. Sistemas de equações diferenciais parciais difusivas, acopladas por termos fonte constituídos por fluxos, como as de Luikov, podem ser adequadamente tratadas pelo esquema Flux-Spline, uma vez que o mesmo se baseia na hipótese de que os fluxos variam de forma linear ao longo dos volumes de controle. Devido ao seu desempenho, pode-se utilizar malhas constituídas por um número menor de volumes de controle que aquelas necessárias ao esquema de diferença central, configurando-se dessa forma, menor esforço computacional para problemas multidimensionais transientes.

\section{LITERATURA CITADA}

Luikov, A.V. Heat and mass transfer in capillary porous bodies, Oxford: Pergamon Press, 1966. 253p. 
Luikov, A.V. Systems of differential equations of heat and mass transfer in capillary porous bodies (Review). International Journal of Heat and Mass Transfer, v.18, p.1-14, 1975.

Luikov, A.V.; Mikhailov, A.Y. Theory of energy and mass transfer. Oxford: Pergamon Press, 1965. 245p.

Nieckele, A.O. Development and evaluation of numerical schemes for the solution of convection-diffusion equations.. Minnesota: University of Minnesota, 1985, 296p. PhD Thesis

Oliveira, P.C. Esquema FLUX-SPLINE aplicado em cavidades abertas com convecção natural. Campinas: UNICAMP, 1997. 196p. Tese Doutorado

Oliveira, P.C. Esquema FLUX-SPLINE aplicado a problemas difusivos tridimensionais em regime permanente. Revista Brasileira de Engenharia Agrícola e Ambiental, Campina Grande, v.3, n.3, p.327-330, 1999.
Patankar, S.V. Numerical heat transfer and fluid flow. New York: Hemisphere Publishing Corporation, 1980. 197p.

Ribeiro, J.W.; Cotta, R. M.; Mikhailov, M. D. Integral transform solution of Luikov's equations for heat and mass transfer in capillary porous media. International Journal of Heat and Mass transfer, v.36, n.18, p.4467-4475, 1993.

Ribeiro, J.W.; Lobo, P.D.C. Drying in capillary porous media. The integral transform method in thermal and fluids science and engineering. New York: Begell House, Inc., cap.7, 1998, 430p.

Varejão, L.M.C. FLUX-SPLINE method for heat, mass and momentum transfer. Minnesota: University of Minnesota, 1979, 235p. Ph. D. Thesis 